\title{
The Social Implications of an Assisted Living Reminder System
}

\author{
Bedoor K. AlShebli, Eric Gilbert, Karrie Karahalios \\ \{alshebli, egilber2, kkarahal\}@cs.uiuc.edu
}

\begin{abstract}
We present the findings of an in situ field study conducted using our assisted living system, I-Living, that aims to enable seniors to live in a costeffective manner independently. Basing the study on both interviews and diaries provided valuable and well-rounded data. Some of the main findings revealed that seniors will wear small health sensors if designed carefully. The study further reveals that delicate and complicated social structures influence the design space in such communities. The primary contribution of this paper is the pilot study conducted at an assisted living facility. It paints a compelling picture of day-to-day life in a healthcare institution and uncovers broad design implications that apply to a wide range of technologies.
\end{abstract}

Keywords: assisted living, pilot study, seniors, reminder system

\section{Introduction}

Americans are living longer than ever before and want to enjoy their senior years with dignity and independence. Today, however, many people spend these years in healthcare facilities with less control over their daily routines. Developing systems that provide remote monitoring and diagnosis would free many people from institutions, thereby reducing financial costs and the need for constant supervision.

In this paper, we present the findings of a pilot study at an assisted living facility. By pairing interviews with diaries, our study contributes a compelling picture of life in a healthcare institution. We describe the social aspects and draw design implications for wearable health sensors and alert systems. In addition, we show designs of interfaces modeled on implications drawn from the study. Therefore, our primary contribution is the pilot study, as it uncovers broad design implications that apply to a wide range of technologies and benefits other practitioners in the field.

Our system, I-Living, [18], ultimately belongs in the home. However, to study its potential impact we focused on people who currently live in facilities but would live at home if given the option. I-Living currently acts as an alert system. It sends seniors reminders, such as medication and doctors' appointments. Its design, however, allows a variety of sensor devices and interfaces to come online via a flexible architecture.

The main goal of our project is to provide the elderly with the option of living independently with confidence and security. When fully realized, we hope I-Living will permit seniors to manage their own health at home in a cost-effective way. The work we present here is the first step towards achieving that goal.

In the remainder of this paper, we discuss related work and its impact on our designs. We then draw a detailed picture of the living environment. We report our findings, concentrating on reminders and forgetfulness. Finally, we conclude with lessons learned from the study and future work informed by these lessons. 


\section{Related Work}

The development of technologies for seniors is becoming increasingly popular. Many companies and universities are pursuing this field. For instance, in the Information Technology for Assisted Living at Home project [2] at the University of California at Berkeley, each user has his/her own Home Health System to monitor the health of the patient.

The Broadband Institute Residential Laboratory of the Aware Home Research Initiative (AHRI) [1] at Georgia Institute of Technology, is a three-story, 5040square-foot home that functions as a living laboratory with the state-of-the-art facilities for broadband access and computing. Projects such as the Digital Family Portrait, [10], and Peek-A-Drawer,[16], were built to improve social relations between family members far apart. Other projects funded by the Aware Home, such as Memory Mirror [11], Cook's Collage [17] and InfoCanvas [9] focus on technologies designed to enable seniors to age in place and simplify their home and activity management.

The PlaceLab [8], a joint MIT and TIAX initiative, is a residential condominium installed with sensing components to help control and monitor activity in the environment. It has been designed to accommodate research into the viability and acceptance of wearable, physiological medical monitoring equipment and their related interfaces, including EKG, pulse oximetry, blood pressure, respiratory auscultation, weight, blood sugar levels, etc., in the context of daily living.

In [4], William Gaver examines different ways to keep the aging population closer to their local community. We look at some of our interfaces as complementing Gaver's efforts by focusing on finding ways to strengthen the relationship and interaction between seniors and their family and friends.

Ken Go's Familyware [5] is another way of reducing the social gap between small communities. However, it does not facilitate text-based, video, or audio mediated means of communication; rather, it supports sharing the "feeling of connection" by sharing objects, with simple signals, such as a child hugging or shaking a teddy bear.

Catherine Plaisant and Ben Schneiderman's personal medical history visualizations, Lifelines [13], inspired some of our more generic clinician interface designs, discussed in the future work section of the paper.

UbiMon [19], at the Imperial College London, addresses issues related to seniors using wearable and implantable sensors for distributed mobile monitoring. Implantable biosensors are used for post-surgical care and monitoring. These sensors can generally be placed inside the body during the operation with minimal additional cost.

Intel's Proactive Health lab [3] also has numerous projects aimed to help and study the needs of seniors dealing with cognitive decline, cancer, and cardiovascular disease.

The Center of Aging Services Technologies, [15], is a website that keeps track of ongoing technologies related to improving the aging experience and healthcare in the US. Also, other related projects are Philip's Personal Health Care Systems [12], British Telecom's House Care [14], and IBM's Personal Care Connect (PCC) [6].

The list goes on. However, due to lack of space we have decided to limit this section to only the universities and companies whose research is closely related to what we're doing and would benefit us in our research. As far as we could tell, we are one of very few to perform an in situ pilot study at an assisted living facility. 


\section{Method and Research Setting}

We conducted semi-structured, one hour pre-interviews and post-interviews with 12 residents and 3 nurses in an assisted living facility. The interviews focused on the two key aspects: wearable health sensors and alert systems. The results of the preinterview informed the design of out interfaces in the facility. The post-interview gave us feedback about the users' impressions of our technology.

To supplement the interviews, we supplied each participant with a diary. Participants recorded their thoughts and impressions when they felt it was appropriate. Diaries complement interviews by offsetting the effects of observers in an interview setting. In our case, the diaries enabled us to gain data that participants would have been reluctant to provide in an interview due to social pressures.

\subsection{The Living Environment}

Our pilot study took place at a working assisted living facility, in a large city in the Midwest, selected for its friendly and helpful atmosphere. We will refer to the facility as St. Ambrose 1 . It sits on a lush, green campus and serves about 140 residents. Residents stroll the grounds multiple times a day. A small chapel sits at the heart of the facility where residents attend the services held every morning and evening. In the afternoons, residents often gather in the community room to read organize activities or just chat over coffee. At mealtime, residents eat together in a large dining room.

The floor where a resident lives indicates their level of independence. The closer a resident lives to the nurses' station, the less independence they get. Residents living on the further floor often guard their independence closely, and tend to cover up each other's falls in the shower, since a bad fall could mean a move closer to the nurses.

\subsection{The Residents}

Most of the residents of St. Ambrose, and all of our participants, are retired nuns. The age of the residential participants ranged from 75 to 94 , with an average age of 88. Most of our participants had spent some time teaching in a Catholic school, 1 participant spent 40 years as a nurse, and 3 participants had dedicated their careers to teaching hearing impaired students. Almost all of our participants hold a Masters degree. All of them demonstrated diligence and interest in the study. One participant called an author 5 days in a row to make sure that the system functioned properly. The residents continually impressed us with their articulate feedback. In one instance, a participant critiqued our interface from the perspective of a deaf person for over 30 minutes, and ended up connecting us by phone with an expert within St. Ambrose.

Some might argue that our participants do not represent the average target user. They do, however, represent the realities of doing field work. Their intelligence, enthusiasm, and insight have helped them provide us with much better feedback than expected. Such ability represents a significant strength of the participants in this study. Often a participant would not only tell us how the technology affected their life, but they would also analyze it from the perspective of other seniors with other disabilities. This served as a great aid at such an early stage in the design phase. Further research is required in this area to understand the diversity of user needs. Still, our study represents a significant first step in this design space.

\footnotetext{
${ }^{1}$ We have changed the name of the facility to protect the identities of our participants.
} 


\subsection{The Nurses}

Three nurses participated in our study. Their years of experience ranged from 3 to 20 . Most of the time, one nurse serves all 140 residents. Therefore, they are usually very busy. During one 30 minute interview, an author counted 9 interruptions. Each interruption seemed to require a different skill from the nurse. In one case, a nurse in a different wing needed a reading about a resident. In another, St. Ambrose's director wanted to talk about a community event in the works. After the $8^{\text {th }}$ interruption, the nurse apologized again and said, "It's always like this around here."

The nurses work in a small nurses' station. They use the station to prepare medication, store resident files and maintain awareness of the ongoing work. During a shift change, the outgoing nurse spends about 15 minutes telling the incoming nurse about the shift's major events. Most of these events revolve around the health of one of the residents. They discuss facts over a thick black binder that the nurses use to log their shifts. Soon after entering the nurses' station, we noticed that the nurses did not use a computer. In fact, a copier was the only electronic device visible in the room.

\subsection{Existing Services}

St. Ambrose is a very tight-knit community and residents often look out for one another. For example, a resident organizes a weekly car pool to the doctor's office, seeks out who should attend, and makes sure that everyone makes their appointment.

In terms of technology, St. Ambrose uses the intercom system the most. Nurses often communicate directly with residents by opening a channel to their room. They can access a resident's room by intercom at any time without having to ask the resident for permission. The facility's staff also uses the intercom to broadcast messages. Twice a day, a priest uses the intercom system to broadcast a prayer.

The nurses use the intercom to remind residents of their medication. If a resident forgets, the intercom is used again. If that fails, the nurse goes to the resident's room.

\section{Pilot Study Pre-Interview}

\subsection{Residents}

Before conducting the pre-interview, state law required us to assess each resident's cognitive ability. To accomplish this, we conducted a Short Blessed Test with each resident [7]. The Short Blessed Test is a six-item test derived from the 26-item Blessed Test developed in 1968. It identifies cognitively impaired patients. The test asks residents to name the year, count backwards from 20 and recall a phrase from memory. All 12 residents passed the test and therefore continued with the study.

Participants were asked about their familiarity with technology. Surprisingly, only 2 of the 12 residents expressed some level of unease with technology.

\subsection{Reminders at St. Ambrose}

The staff at St. Ambrose already provides a set of routines to remind residents to accomplish certain tasks. A chime on the intercom system, for instance, reminds residents of mass. One resident commented, "They really monitor people so they don't forget." The facility established a rule stating that a resident gets one chance to prove 
they can self-medicate upon first entering. They are given two weeks of unsupervised access to their medication. If all goes well, they are allowed to self-medicate. If they fail the test, St. Ambrose takes control of their medication schedule.

However, during the interviews, participants voiced their concern about various situations or events in which they are failed to be reminded of. We categorized these situations as follows and addressed them when designing our interfaces:

- Time: Residents reported needing reminders for meals, meetings, and even the date. One participant even said that some residents will consider you a part of the staff if you can recall the date and time of day.

- Things: Participants confessed to misplacing their belongings regularly. One resident was known to often leave her walker at strange places around the facility.

- Activities: Some noted that they often forget to sign out of the facility or even check their voice-mail. Others forget trips, carpool events, and mundane activities like turning the lights off and wearing clean clothes. In one extreme case, a participant told us of a resident who unknowingly ate 3 pancakes and 4 muffins.

- Place: Residents often forget certain locations, such as their rooms or the chapel.

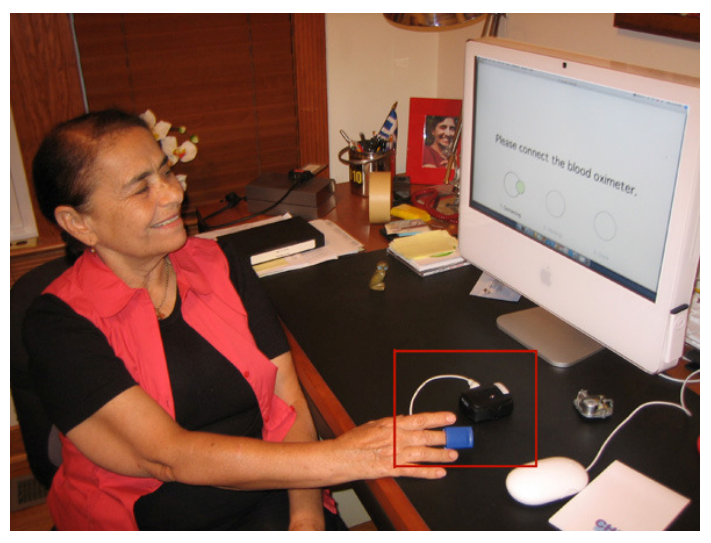

(a)

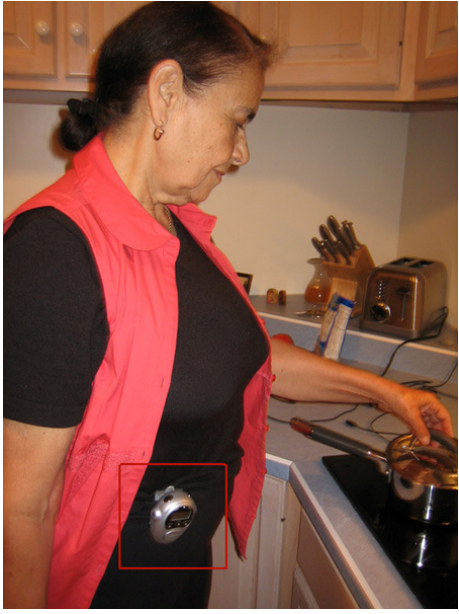

(b)

Figure 1. (a) A resident using the blood oximeter and the vital measurement reminder interface. (b) A resident wearing the placeholder device.

\section{Interface Deployment and Interaction}

Due to limited resources, we provided 2 of the residents with blood oximeter devices, computers and wireless networking (Figure 1(a)). The other 10 participants used placeholder objects that mimicked the dimensions of the blood oximeter sensor. The study ran for 2 weeks with the intention of using low-fidelity technology to gather preliminary data. Using the results, we intend to iterate our current interface designs, create new interfaces and perform a longer and more thorough study. 


\subsection{Reminder Interfaces}

Currently, our system acts as an alert system. The nurses fill out an electronic form (Figure 2(a)) that schedules residents' reminders, such as check-up appointments, medication times and vital measurement readings throughout the day.

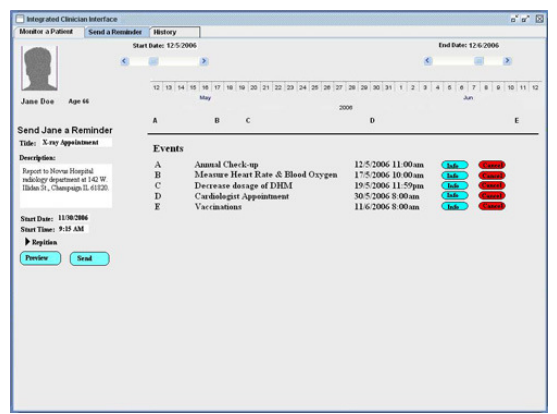

(a)

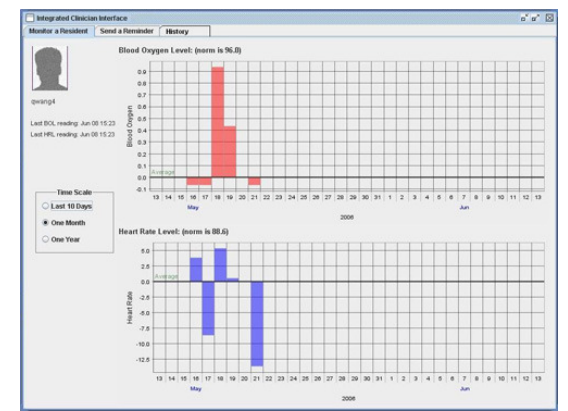

(b)

Figure 2. (a) "Send a Reminder" tab on the clinician's interface. (b) Clinician's user interface showing the two vital sign measurements, blood oxygen and heart rate

We began by modeling the interface after a regular prescription slip. The idea behind it was to ease the transition from the physical to the virtual world. However, a historical account was added to prevent errors like multiple reminders, along with a time line to enable the quick review of assigned reminders.

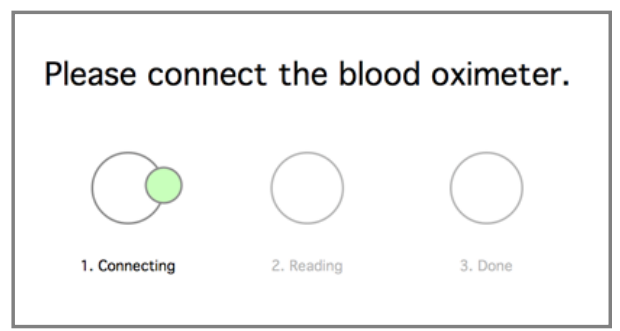

Figure 3. Resident's vital measurement reminder interface.

When a resident receives a reminder on her PC, as shown in Figure 1, she clips the blood oximeter on her finger. An interface (Figure 3) provides residents with directions and feedback throughout the three phases of the reading: connecting, reading and done. The nurses can then monitor the vital signs taken by the residents. The periodic readings submitted by the residents are retrieved from the ALSP (Assisted Living Service Provider) server and displayed in a two-way bar graph (Figure 2(b)). Nurses can view the data at three different time scales: the last 10, 30, or 365 days. The graph is used to display the readings, with the origin being the vital sign norm of the resident. The norm is provided by the resident's physician. By looking at the graphs, the nurses can immediately see whether the resident's heart rate level is above or below the norm and by how much. Should the readings suggest any abnormal behavior, nurses can issue appropriate medical instructions to the resident. 


\subsection{Placeholder Objects}

We gave the remaining ten residents off-the-shelf pedometers, (Figure 1(b)) to act as placeholder devices. They wore them in the morning and took them off before bed. Our aim was to study where and how they wore the devices. We wanted to gauge the residents' reaction to its form and collect data regarding their everyday use. Residents were encouraged to modify the pedometers in any way they saw fit.

\section{Results}

\subsection{Lessons Learned}

After the two week study, post-interviews were conducted with the participants and the diaries were collected. Using the data gathered, we deduced the following:

- Residents were willing to wear the sensing apparatus, as long as it is unobtrusive.

Nine of the ten residents wore the placeholder devices every single day of the two week study. One of the residents dropped hers in water and stopped wearing it for fear of compromising the study. We asked them to wear them however they saw fit. One resident fashioned the device into a necklace, while another fashioned it into a watch. However, we found that most wore them inside a pocket and wished they were thinner. One said, "For the first two days that I wore the instrument, I was wearing a dress and a jacket. The instrument was bulky in the pocket of my jacket; I thought it was unsightly bulging that way. I was thinking how convenient it would be if tit was designed in a rectangle shape and was thin, then it would not bulge" On the other hand, other residents feared losing the device because it was small.

Many participants used the pedometer's text-to-speech features, as well, which reported steps taken, calories, etc. Several reported that the placeholder device made noise at inappropriate times. One resident said, "I named my placeholder Josephine. It felt heavy and clumsy in my pocket. I would have liked it narrower.... Josie talked to me in church."

- There is a fear of breaking or dropping devices.

We found that some would leave the devices in their rooms when leaving the facility. Two dropped their devices in water. One let it dry and continued to use it as normal, while the other dropped out for fear of compromising the study. In a diary statement, a resident reported issues with wearing the device, saying: "Even though it did not fall off my slacks band, I did not wear it for more than a half-day. Because I have a bladder disease, I use the rest room frequently and found it to be bothersome to be concerned each time the instrument would fall. ... On Fri. (June 23rd) I wore a jacket with shallow pockets. I placed the instrument in the pocket; it fell when I used the rest room and when I was having breakfast."

- The monitoring interface was intuitive and encouraged further exploration.

The two residents using the monitoring interface found the interface useful and very easy to use. They were very articulate in describing their experiences. Based on that, it was decided that the next version of the resident monitoring device will be a screen against a wall that provides alerts and shows pictures of their choosing at other times. This decision was a result of several interviews with one of the 
residents using the working device. "Could you make it into a bed or chair side picture that's a screensaver of a relative. Don't make it a glaring outside object."

The interface was created to be simple and not require any keyboard use by the residents. However, we found them wanting to delve further into their own data and willing to use a keyboard or touch screen. Also, more residents wanted to use the working prototype. In the future, we hope to equip more residents with working systems and with more devices such as scales and blood pressure units.

None of the residents were comfortable using a cell phone. Similarly, many felt the buttons on the placeholder objects were small and difficult to read. One resident mentioned that arthritis kept her from using the pedometer and asked to have the battery removed. Furthermore, the system was not fully trusted and at times did not go off when expected. One said, "One went off at 11:45 p.m., and I was forced to get up. Is there someone in my room? Maybe a reason not to use a human voice at night" This was due to wireless networking glitches the first few days of the study.

- Nurses want an interface that monitors more vital signs and provides privacy.

The nurses' primary concern was displaying information they normally checked, such as blood pressure. Also, they were very busy with so many residents that privacy during discussions was a large complaint by the residents.

\subsection{Social Factors Learned}

During our short stay at St. Ambrose, we came to realize the existence of a very strong social network amongst the residents. For instance, the residents did not want reminders for every possible medication they should take. Some of them enjoy going to the nurse's station for medication and anticipate the social aspect of the gathering. Car pooling for doctor appointments was another time where the residents enjoyed socializing and catching up. Every Sunday, all the sisters went to mass and would socialize afterwards while waiting their turn to take the elevator. Furthermore, the residents try to organize a social event every afternoon or night, such as Friday night movies. Therefore, any engineering approach targeted at assisted living residents should reflect on these social aspects.

\section{Future Work}

\subsection{Future Visualizations}

Only one nurse is usually on duty at time. Due to that, the nursing staff are always overwhelmed. One of their main concerns with our system was that it might end up taking up even more of their time. They weren't very interested in the blood oximeters and didn't want data that wasn't in their job description. They wanted information they actually needed and would help save trips to residents rooms. Upon listening to these concerns, we decided to design two visualizations that could help them. One is to view the resident's health history and the other to show their current status at a glance.

Medical History's Visualization. This visualization (Figure 4(a)), inspired by Ben Schneiderman's Lifelines [13], is designed to help clinicians/nurses understand the resident's background/medical history in a timely manner. It shows the resident's 
medical history over a period of time and divides it into five main sections: Illnesses, Medications, Doctors, Interventions, and Hospitalization. By scrolling the bars on the top, the clinician can go back and forth in history to see the different times of illnesses.

Current Position and General Health Visualization. This visualization (Figure 4(b)) would help clinicians, family and friends check on the residents in a non timeconsuming manner. It shows two main parameters:

- the position of the user at the current time, shown by the angle at which the resident's name appears in the visualization.

- the general health condition of the user at the current time, shown by location of the resident's name on the radius of the visualization. In other words, the closer the name is to green, the better their overall health condition is, and vice versa.

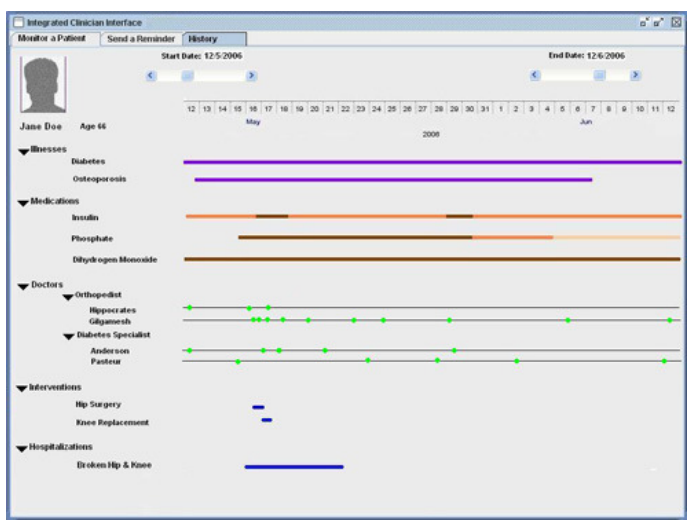

(a)

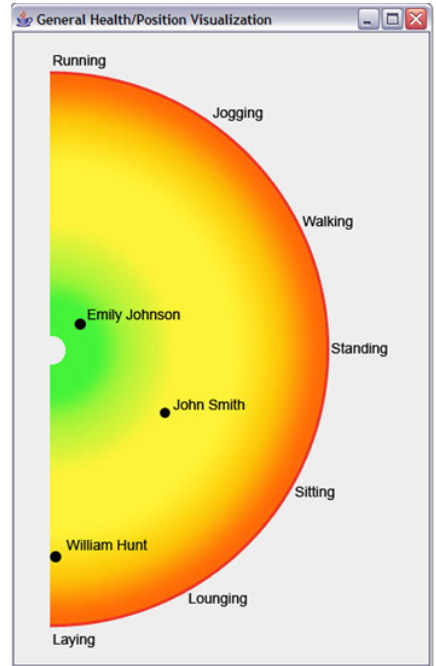

(b)

Figure 4. (a) Resident Medical History visualization. (b) Position/General Health visualization

\subsection{Future Devices}

Based on the social implications learned from this study, we found that some of the residents might be more accepting and comfortable in using devices that they have already are or have been using since their youth. They also wouldn't mind wearing any devices that might be common among a younger generation. Therefore, we hope to introduce devices that can be used by all ages, which would monitor health amongst other things.

\subsection{Future User Studies}

In the future, we hope to conduct a longer and more thorough ethnography. We hope to use a different set of users that might better represent what our average target user might be. Three facilities have already agreed to participate in our studies. The 
participants are closer to typical targeted end users with an average age of 78 and a more even gender distribution (55\% female and $45 \%$ male). A more robust network will be installed using wireless range extenders, and more sensors will be used. Finally, we hope to install most, if not all, of the interfaces mentioned in this section.

\section{References}

1. Abowd, G, Price, E. AwareHome: Georgia Institute of Technology: http://www.awarehome. gatech.edu/

2. Bajcsy, R. ITALH: The Information Technology for Assisted Living at Home project. University of California at Berkeley. http://www.eecs.berkeley.edu/ eklund/projects/ITALH/

3. Dishman, E. Proactive Health Laboratory. Intel. http://www.intel.com/research/prohealth/

4. Gaver, W.W., and Dunne A. (1999): Project Realities: Conceptual Design for Cultural Effect. Proceedings of ACM CHI'99 Conference on Human Factors in Computing Systems, 600-607

5. Go, K., Carroll, J.M., Imamiya, A.: Familyware: communicating with someone you love. Proceedings of HOIT: Home-Oriented Informatics and Telematics 2000.

6. Husemann, D. PCC: Personal Care Connect. IBM. http://www.zurich.ibm.com/pcc/

7. Katzman R., Brown T., Fuld P., Peck A., Schechter R., Schimmel H.: Validation of a short Orientation-Memory-Concentration Test of cognitive impairment. Am J Psychiatry. 1983.

8. Larson, K., Intille, S. The PlaceLab. http://architecture.mit.edu/house_n/placelab.html

9. Miller, T. and Stasko, J.: The InfoCanvas: information conveyance through personalized, expressive art. CHI '01 extended abstracts on Human factors in computing system.

10. Mynatt, E. D., Rowan, J., Craighill, S., and Jacobs A.: Digital family portraits: supporting peace of mind for extended family members. CHI '01: Proceedings of the SIGCHI conference on Human factors in computing systems.

11. Mynatt, E. D. Memory Mirror. http://www-static.cc.gatech.edu/fce/ecl/projects/dejaVu/mm/ index.html

12. Philips. Personal Health Care Systems: http://www.extra.research.philips.com/swa/cluster _phcs.html

13. Plaisant C., Milash, B., Rose, A., Widoff, S., and Schneiderman, B. : LifeLines: visualizing personal histories. CHI '96: Proceedings of the SIGCHI conference on Human factors in computing systems. Vancouver, British Columbia, Canada.

14. Porteus, J., Brownsell, S. House Care. British Telecom. http://www.housingcare.org/ downloads/kbase/2334.pdf

15. Scritchfield, R. CAST: Center of Aging Services Technologies,. http://www.agingtech.org.

16. Siio, I., Rowan, J., and Mynatt, E. : Peek-a-drawer: communication by furniture. CHI '02 extended abstracts on Human factors in computing systems. Minneapolis, Minnesota, USA.

17. Tran, Q., Calcaterra, G., Mynatt, E. : Cook's Collage: Deja Vu Display for a Home Kitchen. Proceedings of HOIT: Home-Oriented Informatics and Telematics 2005, 15-32.

18. Wang Q., Shin W., Liu X., Zeng Z., Oh C., AlShebli B. K., Caccamo M., Gunter C. M., Gunter E., Hou J., Karahalios K., and Sha L. I-Living: An Open System Architecture for Assisted Living. Proceedings of IEEE SMC 2006.

19. Yang, G. UbiMon: the Ubiquitous Monitoring Environment for Wearable and Implantable Sensors. Imperial University London. http://www.doc.ic.ac.uk/vip/ubimon/home/index.html 